\title{
Covariance systems
}

\author{
Jan Naudts and Maciej Kuna* \\ Departement Natuurkunde, Universiteit Antwerpen UIA \\ Universiteitsplein 1, B-2610 Antwerpen, Belgium \\ Email: naudts@uia.ua.ac.be, kuna@uia.ua.ac.be
}

v6, September 2000

\begin{abstract}
We introduce new definitions of states and of representations of covariance systems. The GNS-construction is generalized to this context. It associates a representation with each state of the covariance system. Next, states are extended to states of an appropriate covariance algebra. Two applications are given. We describe a nonrelativistic quantum particle, and we give a simple description of the quantum spacetime model introduced by Doplicher et al [10, 11].
\end{abstract}

KEYWORDS: covariance system, quantum spacetime, GNS-representation, noncanonical commutation relations, projective representations, $C^{*}$-multipliers

\section{Introduction}

Consider an action $\sigma$ of a group $X$ as automorphisms of a $C^{*}$-algebra $\mathcal{A}$. A *-representation $\pi$ of $\mathcal{A}$ is $X$-covariant if a unitary representation $U$ of $X$ exists such that

$$
\pi\left(\sigma_{x} a\right)=U(x) \pi(a) U(x)^{*}
$$

*On leave from the Technical University of Gdansk 
holds for all $a \in \mathcal{A}$ and $x \in X$. A state $\omega$ of $\mathcal{A}$ is $X$-covariant if its GNSrepresentation is $X$-covariant. Doplicher et al [5] proved a one-to-one relation between covariant $*$-representations of $\mathcal{A}$ and $*$-representations of the crossed product algebra $\mathcal{A} \times_{\sigma} X$. In quantum mechanics also projective representations of the relevant symmetry group $X$ are important. A *-representation $\pi$ of $\mathcal{A}$, which is only covariant in the sense that (1) holds w.r.t. a projective representation $U$ of $X$, cannot extend to a *-representation of $\mathcal{A} \times_{\sigma} X$ because then $U$ would not be projective. This leads to the situation that the covariance algebra $\mathcal{A} \times{ }_{\sigma} X$ has to be replaced by some other algebra which depends in general on the choice of $\pi$. An elegant formalism to deal with this situation is presented here. It introduces new concepts of representation and state of a covariance system. The GNS-construction, well-known for states of $C^{*}$-algebras, is generalized and associates a representation with each state of the covariance system. Next, it is shown that each state of the covariance system extends to a state of an appropriate covariance algebra.

Our results on covariance systems combine well with a new $C^{*}$-algebraic approach to quantum mechanics and quantum field theory. The quantum mechanics of a single nonrelativistic particle is described by the covariance system consisting of the group of spatial shifts $\mathbf{R}^{3},+$, acting on an abelian algebra of functions of position. If projective representations are allowed then the group of shifts can be replaced by the full Galilei group. Indeed, LevyLeblond [4] has shown, using results of Bargmann [2], that the physically relevant representations of the Galilei group are projective representations, labeled by a free parameter, which is proportional to the mass of the particle. In addition, projective representations of the subgroup of rotations describe the spin of the particle. One concludes that, in the terminology of the present paper, each nonrelativistic particle is described by a state of the covariance system. It has a mass, and can have a spin.

As a second example we consider the model of quantum spacetime introduced by Doplicher et al [10, 11]. We restrict ourselves to the description of a single particle. Its configuration space $\Sigma$ is a 4 -dimensional manifold in $\mathbf{R}^{6}$. Shifts in Minkowski space and in momentum space act on $\Sigma$ in the trivial way. An explicit expression is given for a quasifree state of the resulting covariance system. It involves a $C^{*}$-multiplier with values in the algebra of continuous complex functions of $\Sigma$. It has a nontrivial GNS- representation in which the shift groups have projective representations. Their generators are position and momentum operators satisfying noncanonical commutation relations. 
A third example, the electromagnetic radiation field, will be discussed elsewhere.

\section{Covariance systems}

\section{$1.1 \quad C^{*}$-multipliers}

The following definition introduces a pair of objects $(\xi, \sigma)$, where $\xi$ generalizes the concept of multiplier to maps with values in a $C^{*}$-algebra, and where $\sigma$ is a twisted representation of a group as automorphisms of a $C^{*}$-algebra. Operator valued multipliers have been studied in [6, 12].

Definition 1. A (left) $C^{*}$-multiplier of a locally compact group $X$ with associated twisted representation $\sigma$ is a measurable map $\xi$ of $X \times X$ into the unitary elements of the multiplier algebra $M(\mathcal{A})$ of a $C^{*}$-algebra $\mathcal{A}$ satisfying

$$
\xi(x, e)=\xi(e, y)=\mathbf{I}, \quad x, y \in X
$$

together with a map $\sigma$ of $X$ into the automorphisms of $\mathcal{A}$ such that $\sigma_{e}$ is the identity transformation and

$$
\sigma_{x} \xi(y, z)=\xi(x, y) \xi(x y, z) \xi(x, y z)^{*}, \quad x, y, z \in X
$$

and

$$
\sigma_{x} \sigma_{y} a=\xi(x, y)\left(\sigma_{x y} a\right) \xi(x, y)^{*}, \quad x, y \in X, a \in \mathcal{A}
$$

Throughout the paper we assume that $\xi$ is continuous in a neighborhood of the neutral element $e$ of $X$. If $\xi(x, y)$ is a multiple of $\mathbf{I}$ for all $x, y \in X$ then $\xi$ is also called a cocycle.

A right $C^{*}$-multiplier $\zeta$ satisfies

$$
\sigma_{y}^{-1} \zeta(z, x)=\zeta(z x, y)^{*} \zeta(z, x y) \zeta(x, y), \quad x, y, z \in X
$$

instead of (3) and

$$
\sigma_{x} \sigma_{y} a=\sigma_{x y}\left(\zeta(x, y) a \zeta(x, y)^{*}\right), \quad x, y \in X, a \in \mathcal{A}
$$

instead of (1). 


\subsection{Covariant states}

The following definition can be found in the literature, see e.g. [9].

Definition 2. A covariance system is a triple $(\mathcal{A}, X, \sigma)$ consisting of a $C^{*}$ algebra $\mathcal{A}$, a locally compact symmetry group $X$, and a continuous action $\sigma$ of $X$ as automorphisms of $\mathcal{A}$.

The definition generalizes that of a dynamical system as often found in the literature (take $X=\mathbf{R},+$, and $t \in \mathbf{R} \rightarrow \sigma_{t}$ the time evolution of the quantum system). Here we have rather different applications in mind. Examples are given further on.

The next definition is new.

Definition 3. A state of the covariance system $(\mathcal{A}, X, \sigma)$ is a measurable map $\omega$ of $X \times X$ into the continuous linear complex valued functions of $\mathcal{A}$, having the following properties.

- (positivity) For all $n>0$ and for all possible choices of $\lambda_{1}, \ldots, \lambda_{n}$ in $\mathbf{C}$, of $x_{1}, \ldots, x_{n}$ in $X$, and of $a_{1}, \ldots a_{n}$ in $\mathcal{A}$ is

$$
\sum_{j, k=1}^{n} \lambda_{j} \overline{\lambda_{k}} \omega_{x_{j}, x_{k}}\left(a_{k}^{*} a_{j}\right) \geq 0
$$

- (normalization) $\omega_{e, e}$ is a state of $\mathcal{A}$.

- (covariance) There exists a right $C^{*}$-multiplier $\zeta$ of $X$ with values in the multiplier algebra $M(\mathcal{A})$ of $\mathcal{A}$ such that

$$
\omega_{x, y}\left(\sigma_{z} a\right)=\omega_{x z, y z}\left(\zeta(y, z) a \zeta(x, z)^{*}\right)
$$

for all $x, y, z \in X$ and $a \in \mathcal{A}$.

- (continuity) For any $a \in \mathcal{A}$ the map $x, y \rightarrow \omega_{x, y}(a)$ is continuous in a neighborhood of the neutral element of $X$.

The state $\omega$ is faithful if $\omega_{e, e}$ is faithful.

Any $X$-covariant state $\omega$ of $\mathcal{A}$ defines a state (again denoted $\omega$ ) of the covariance system $(\mathcal{A}, X, \sigma)$ by

$$
\omega_{x, y}(a)=\left(\pi(a) U(x)^{*} \Omega, U(y)^{*} \Omega\right), \quad x, y \in X, a \in \mathcal{A}
$$


where $(\mathcal{H}, \pi, \Omega)$ is the GNS-representation of $\mathcal{A}$ induced by $\omega$, and $U$ is the covariant representation of $X$. The converse is also true. If $\omega$ is a state of $(\mathcal{A}, X, \sigma)$ then $\omega_{e, e}$ is an $X$-covariant state of $\mathcal{A}$, provided we weaken the definition of $X$-covariance to allow for projective representations. This will be shown in section 1.5 .

The following results are needed for technical reasons. The next lemma generalizes the lemma of Schwarz.

Lemma 1. Let $\omega$ be a state of $(\mathcal{A}, X, \sigma)$. Then

$$
\left|\sum_{j, k=1}^{n} \lambda_{j} \overline{\lambda_{k}} \omega_{x_{j}, x_{k}}\left(a_{k}^{*} b_{j}-b_{k}^{*} a_{j}\right)\right|^{2} \leq 4 \sum_{j, k=1}^{n} \lambda_{j} \overline{\lambda_{k}} \omega_{x_{j}, x_{k}}\left(a_{k}^{*} a_{j}\right) \sum_{j, k=1}^{n} \lambda_{j} \overline{\lambda_{k}} \omega_{x_{j}, x_{k}}\left(b_{k}^{*} b_{j}\right)
$$

for all choices of $\lambda_{1}, \ldots, \lambda_{n}$ in $\mathbf{C}$, of $x_{1}, \ldots, x_{n}$ in $X$, and of $a_{1}, \ldots a_{n}$ and $b_{1}, \ldots, b_{n}$ in $\mathcal{A}$.

The proof of the lemma is straightforward. The lemma is now used to prove uniform continuity of $\omega_{x, y}$.

Proposition 1. One has

$$
\sum_{j, k=1}^{n} \lambda_{j} \overline{\lambda_{k}} \omega_{x_{j}, x_{k}}\left(a_{k}^{*} a_{j}\right) \leq\left(\sum_{j=1}^{n}\left|\lambda_{j}\right||| a_{j}||\right)^{2}
$$

for all choices of $\lambda_{1}, \ldots, \lambda_{n}$ in $\mathbf{C}$, of $x_{1}, \ldots, x_{n}$ in $X$, and of $a_{1}, \ldots a_{n}$ in $\mathcal{A}$.

Proof. The statement is clearly true for $n=1$. Assume it to hold up to $n-1$. One calculates, using the lemma with $b_{j}=i \delta_{k, n} a_{n}$,

$$
\begin{aligned}
\sum_{j, k=1}^{n} \lambda_{j} \overline{\lambda_{k}} \omega_{x_{j}, x_{k}}\left(a_{k}^{*} a_{j}\right) & \leq\left(\sum_{j=1}^{n-1}\left|\lambda_{j}\right||| a_{j}||\right)^{2}+\left|\lambda_{n}\right|^{2} \|\left. a_{n}\right|^{2} \\
& +\sum_{j=1}^{n-1} \lambda_{j} \overline{\lambda_{n}} \omega_{x_{j}, x_{n}}\left(a_{n}^{*} a_{j}\right)+\sum_{j=1}^{n-1} \lambda_{n} \overline{\lambda_{j}} \omega_{x_{n}, x_{j}}\left(a_{j}^{*} a_{n}\right) \\
& \leq\left(\sum_{j=1}^{n}\left|\lambda_{j}\right||| a_{j}||\right)^{2}
\end{aligned}
$$

Hence the proof follows by induction. 


\subsection{Representations of a covariance system}

The following two definitions are obvious.

Definition 4. A representation of the covariance system $(\mathcal{A}, X, \sigma)$ is a triple $(\mathcal{H}, \pi, U)$ which consists of a ${ }^{*}$-representation $\pi$ of $\mathcal{A}$ in a Hilbert space $\mathcal{H}$, and a measurable map $x \in X \rightarrow U(x)$ into the unitary operators of $\mathcal{H}$, with the properties that (1) holds, and that each normalized element $\psi$ of $\mathcal{H}$ defines an $X$-covariant state $\omega$ by (9). An element $\psi \in \mathcal{H}$ is cyclic for the representation if the subspace spanned by

$$
\left\{\pi(a) U(x)^{*} \psi: a \in \mathcal{A}, x \in X\right\}
$$

is dense in $\mathcal{H}$.

Definition 5. Two representations $(\mathcal{H}, \pi, U)$ and $\left(\mathcal{H}^{\prime}, \pi^{\prime}, U^{\prime}\right)$ of $(\mathcal{A}, X, \sigma)$ are equivalent if there exists an isomorphism $V$ of $\mathcal{H}$ onto $\mathcal{H}^{\prime}$ intertwining $\pi$ and $\pi^{\prime}$, resp. $U$ and $U^{\prime}$.

\subsection{Projective representations of $X$}

Proposition 2. Let $\pi$ be $a^{*}$-representation of $\mathcal{A}$ in a Hilbert space $\mathcal{H}$ and $x \rightarrow U(x)$ is a projective representation of $X$ in $\mathcal{H}$ in the sense that $U(e)=\mathbf{I}$, $U(x)$ is unitary for all $x \in X$, and $a C^{*}$-multiplier $\xi$ exists with values in the center of $M(\mathcal{A})$ such that

$$
U(x) U(y)=\pi(\xi(x, y)) U(x y), \quad x, y \in X
$$

Assume that the twisted representation associated with $\xi$ coincides with the action $\sigma$ of $(\mathcal{A}, X, \sigma)$, that $x \rightarrow U(x)$ is strongly continuous in a neighborhood of the neutral element of $X$, and that the covariance condition (1) is satisfied. Then $(\mathcal{H}, \pi, U)$ is a representation of the covariance system $(\mathcal{A}, X, \sigma)$.

Proof. Each normalized element $\psi$ of $\mathcal{H}$ defines a covariant state $\omega$ by (9). In particular, one has

$$
U(x y)^{*} U(x) U(y)=\pi(\zeta(x, y))
$$

with

$$
\zeta(x, y)=\sigma_{x y}^{-1} \xi(x, y)
$$


Using this result covariance follows from

$$
\begin{aligned}
\omega_{x, y}\left(\sigma_{z} a\right) & =\left(\pi\left(\sigma_{z} a\right) U(x)^{*} \psi, U(y)^{*} \psi\right) \\
& =\left(\pi(a) U(z)^{*} U(x)^{*} \psi, U(z)^{*} U(y)^{*} \psi\right) \\
& =\left(\pi(a)(U(x z) \pi(\zeta(x, z)))^{*} \psi,(U(y z) \pi(\zeta(y, z)))^{*} \psi\right) \\
& =\omega_{x z, y z}\left(\zeta(y, z) a \zeta(x, z)^{*}\right)
\end{aligned}
$$

The requirement that $\xi(x, y)$ commutes with all elements of $\mathcal{A}$ is needed because the action $\sigma$ of $X$ on $\mathcal{A}$ is not twisted with $\xi$ but is a representation of $X$ as automorphisms of $\mathcal{A}$.

\subsection{GNS-construction}

The GNS-construction for states of a $C^{*}$-algebra can be generalized as follows.

Theorem 1. Let $\omega$ be a covariant state of the covariance system $(\mathcal{A}, X, \sigma)$. There exists a representation $(\mathcal{H}, \pi, U)$ of $(\mathcal{A}, X, \sigma)$ satisfying

- $U$ is a projective representation of $X$ with multiplier $\xi$.

- (8) holds with $\zeta$ related to $\xi$ by (10).

- There exists a cyclic vector $\Omega$ of $\mathcal{H}$ such that (9) holds.

The quadruple $(\mathcal{H}, \pi, U, \Omega)$ is unique up to equivalence of representations, i.e. if $\left(\mathcal{H}^{\prime}, \pi^{\prime}, U^{\prime}\right)$ is a representation and $\Omega^{\prime}$ is a cyclic vector of $\mathcal{H}^{\prime}$ satisfying (9) with $\psi=\Omega^{\prime}$ and $\pi$ and $U$ replaced by $\pi^{\prime}$ and $U^{\prime}$, then there exists an isomorphism $V$ of $\mathcal{H}$ onto $\mathcal{H}^{\prime}$ intertwining $\pi$ and $\pi^{\prime}$, resp. $U$ and $U^{\prime}$, and mapping $\Omega$ onto $\Omega^{\prime}$.

Proof. Let $\mathcal{C}_{c}(X, \mathcal{A})$ denote the linear space of continuous functions with compact support in $X$ and with values in $\mathcal{A}$. A sesquilinear form is defined by

$$
(f, g)=\int_{X} \mathrm{~d} x \Delta(x)^{-1} \int_{X} \mathrm{~d} y \Delta(y)^{-1} \omega_{x, y}\left(g(y)^{*} f(x)\right)
$$

for all $f, g \in \mathcal{C}_{c}(X, \mathcal{A})$ ( $\Delta$ is the modular function of $X$ ). From the positivity of $\omega$ follows that $(\cdot, \cdot)$ is a positive form. Let us assume for simplicity of 
notations that $(\cdot, \cdot)$ is not degenerated (it is easy to see that further definitions do not depend on the choice of the representative of the equivalent class given by the kernel of $(\cdot, \cdot))$. Then it is an inner product making $\mathcal{C}_{c}(X, \mathcal{A})$ into a pre-Hilbert space. Let $\mathcal{H}$ denote its completion.

Define $\pi$ by

$$
\pi(a) f(x)=a f(x), \quad a \in \mathcal{A}, f \in \mathcal{C}_{c}(X, \mathcal{A}), x \in X
$$

By linearity $\pi(a)$ extends to a linear operator with domain $\mathcal{C}_{c}(X, \mathcal{A})$. If $a \geq 0$ then

$$
(\pi(a) f, f)=\int_{X} \mathrm{~d} x \Delta(x)^{-1} \int_{X} \mathrm{~d} y \Delta(y)^{-1} \omega_{x, y}\left(f(y)^{*} a f(x)\right) \geq 0
$$

This implies that $\pi(a)$ is bounded. Since each element of $\mathcal{A}$ is a linear combination of positive elements one concludes that $\pi(a)$ is bounded for all $a \in \mathcal{A}$.

It is obvious that $\pi(a) \pi(b)=\pi(a b)$, that $\pi$ is linear, and that $\pi(b)^{*}=$ $\pi\left(b^{*}\right)$. Hence $\pi$ is a ${ }^{*}$-representation of $\mathcal{A}$ in $\mathcal{H}$.

Next define a linear operator $U$ by

$$
U(x) f(y)=\sigma_{x}[f(y x) \zeta(y, x)] \quad x, y \in X, f \in \mathcal{C}_{c}(X, \mathcal{A})
$$

Note that one has $U(e)=$ I. A straightforward calculation gives

$$
U(x)^{*} f(y)=\left(\sigma_{x}^{-1} f\left(y x^{-1}\right)\right) \zeta\left(y x^{-1}, x\right)^{*}
$$

This expression can be used to verify that $U(x)$ is unitary.

Let $\xi$ be defined by (16). Then a short calculation using (5) gives

$$
\begin{aligned}
U(x) U(y) f(z) & =\sigma_{x}\left[\sigma_{y}[f(z x y) \zeta(z x, y)] \zeta(z, x)\right] \\
& =\sigma_{x y}[f(z x y) \zeta(x, y) \zeta(z, x y)] \\
& =\pi(\xi(x, y)) U(x y) f(z)
\end{aligned}
$$

which is (14).

Let $\left(u_{\alpha}\right)_{\alpha}$ be an approximate unit of $\mathcal{A}$. For each neighborhood $v$ of $e$ in $X$ let $\delta_{v}$ be a positive function vanishing outside $v$ and satisfying

$$
\int_{X} \mathrm{~d} x \delta_{v}(x)=1
$$


Then the functions $\left(\delta_{v} u_{\alpha}\right)_{v, \alpha}$ form a Cauchy sequence in $\mathcal{H}$, converging to some element $\Omega$. From (22) follows now that

$$
\int_{X} \mathrm{~d} x \Delta(x)^{-1} \pi(f(x)) U(x)^{*} \Omega=f
$$

for all $f \in \mathcal{C}_{c}(X, \mathcal{A})$. The latter implies

$$
(f, g)=\int_{X} \mathrm{~d} x \Delta(x)^{-1} \int_{X} \mathrm{~d} y \Delta(y)^{-1}\left(\pi\left(g(y)^{*} f(x)\right) U(x)^{*} \Omega, U(y)^{*} \Omega\right)
$$

Comparison with (18) shows that (9) holds. From (21) and (22) follows immediately that (11) holds. Hence, proposition 2 asserts that $(\mathcal{H}, \pi, U)$ is a representation of the covariance system.

Finally, we prove uniqueness up to equivalence of representations. Define $V$ with domain $\mathcal{C}_{c}(X, \mathcal{A})$ by

$$
V f=\int_{X} \mathrm{~d} x \Delta(x)^{-1} \pi^{\prime}(f(x)) U^{\prime}(x)^{*} \Omega^{\prime}
$$

It is straightforward to verify that $V$ extends to an isometry of $\mathcal{H}$ into $\mathcal{H}^{\prime}$. Because $\Omega^{\prime}$ is cyclic $V$ is an isomorphism.

That $V$ intertwines $\pi$ and $\pi^{\prime}$ is obvious. For $U$ and $U^{\prime}$ one has

$$
\begin{aligned}
U^{\prime}(x) V f & =U^{\prime}(x) \int_{X} \mathrm{~d} y \Delta(y)^{-1} \pi^{\prime}(f(y)) U^{\prime}(y)^{*} \Omega^{\prime} \\
& =U^{\prime}(x) \int_{X} \mathrm{~d} y \Delta(y)^{-1} \pi^{\prime}(f(y x)) U^{\prime}(y x)^{*} \Omega^{\prime} \\
& =U^{\prime}(x) \int_{X} \mathrm{~d} y \Delta(y)^{-1} \pi^{\prime}(f(y x) \zeta(y, x)) U^{\prime}(x)^{*} U^{\prime}(y)^{*} \Omega^{\prime} \\
& =\int_{X} \mathrm{~d} y \Delta(y)^{-1} \pi^{\prime}\left(\sigma_{x}[f(y x) \zeta(y, x)]\right) U^{\prime}(y)^{*} \Omega^{\prime} \\
& =V U(x) f
\end{aligned}
$$

Finally, it is obvious that $V \Omega=\Omega^{\prime}$.

\subsection{Crossed product algebras}

A reader not interested in crossed product algebras may skip this section. It is not needed for the sequel of the paper but is added to clarify the relation 
between the present work and previous work on crossed product algebras. Details found in [12 are not repeated here.

Let be given a state $\omega$ of a covariance system $(\mathcal{A}, X, \sigma)$. Let $\zeta$ be the right multiplier associated with $\omega$. Let $\xi$ be the left multiplier derived from $\zeta$ by $(16)$. The representation $\sigma$ together with $\xi$ determine a crossed product algebra $\mathcal{A} \times_{\xi} X$. It is constructed as follows. Let $\mathcal{L}_{1}(X, \mathcal{A})$ denote the linear space of integrable functions of $X$ with values in $\mathcal{A}$. A product law for elements of $\mathcal{L}_{1}(X, \mathcal{A})$ is given by

$$
(f \times g)(x)=\int_{X} \mathrm{~d} y f(y) \xi\left(y, y^{-1} x\right) \sigma_{y} g\left(y^{-1} x\right)
$$

An involution is given by

$$
f^{\star}(x)=\Delta(x)^{-1} \xi\left(x, x^{-1}\right)^{*} \sigma_{x} f\left(x^{-1}\right)^{*}
$$

In this way $\mathcal{L}_{1}(X, \mathcal{A})$ becomes an involutive algebra. By closure in an appropriate norm it becomes the $C^{*}$-algebra $\mathcal{A} \times{ }_{\xi} X$.

A linear functional $\bar{\omega}$ of $\mathcal{L}_{1}(X, \mathcal{A})$ is defined by

$$
\bar{\omega}(f)=\int_{X} \mathrm{~d} x \Delta(x)^{-1} \omega_{x, e}\left(\xi\left(x^{-1}, x\right) f\left(x^{-1}\right)\right)
$$

Proposition 3. $\bar{\omega}$ extends to a state of $\mathcal{A} \times_{\xi} X$.

Proof. Positivity is verified as follows.

$$
\begin{aligned}
& \bar{\omega}\left(f^{\star} \times f\right) \\
= & \int_{X} \mathrm{~d} x \Delta(x)^{-1} \omega_{x, e}\left(\xi\left(x^{-1}, x\right)\left(f^{\star} \times f\right)\left(x^{-1}\right)\right) \\
= & \int_{X} \mathrm{~d} x \Delta(x)^{-1} \int_{X} \mathrm{~d} y \Delta(y)^{-1} \\
\times & \omega_{x, e}\left(\xi\left(x^{-1}, x\right) \xi\left(y, y^{-1}\right)^{*}\left(\sigma_{y} f\left(y^{-1}\right)\right)^{*} \xi\left(y, y^{-1} x^{-1}\right) \sigma_{y} f\left(y^{-1} x^{-1}\right)\right) \\
= & \int_{X} \mathrm{~d} x \Delta(x)^{-1} \int_{X} \mathrm{~d} y \Delta(y)^{-1} \\
\times & \left.\omega_{x, e}\left(\sigma_{y}\left[\xi\left(y^{-1} x^{-1}, x\right) f\left(y^{-1}\right)\right)^{*} f\left(y^{-1} x^{-1}\right)\right]\right) \\
= & \int_{X} \mathrm{~d} x \Delta(x)^{-1} \int_{X} \mathrm{~d} y \Delta(y)^{-1} \\
\times & \left.\omega_{x y, y}\left(\xi\left(y^{-1} x^{-1}, x\right) \zeta(x, y)^{*} f\left(y^{-1}\right)\right)^{*} f\left(y^{-1} x^{-1}\right)\right)
\end{aligned}
$$




$$
\begin{aligned}
& =\int_{X} \mathrm{~d} x \Delta(x)^{-1} \int_{X} \mathrm{~d} y \Delta(y)^{-1} \\
& \left.\times \omega_{x y, y}\left(\xi\left(y^{-1}, y\right) \xi\left(y^{-1} x^{-1}, x y\right)^{*} f\left(y^{-1}\right)\right)^{*} f\left(y^{-1} x^{-1}\right)\right) \\
& \left.=\int_{X} \mathrm{~d} x \Delta(x)^{-1} \int_{X} \mathrm{~d} y \Delta(y)^{-1} \omega_{x, y}\left(\xi\left(y^{-1}, y\right) \xi\left(x^{-1}, x\right)^{*} f\left(y^{-1}\right)\right)^{*} f\left(x^{-1}\right)\right) \\
& \geq 0
\end{aligned}
$$

For each neighborhood $v$ of the neutral element of $X$ let $\delta_{v}$ be a positive normalized function with support in $v$. Then $\left(\delta_{v} u_{\alpha}\right)_{v, \alpha}$ is an approximate unit of $\mathcal{L}_{1}(X, \mathcal{A})$. One has

$$
\bar{\omega}\left(\delta_{v} u_{\alpha}\right)=\int_{X} \mathrm{~d} x \Delta(x)^{-1} \delta_{v}\left(x^{-1}\right) \omega_{x, e}\left(\xi\left(x^{-1}, x\right) u_{\alpha}\right)
$$

The latter tends to 1 because of continuity of $x \rightarrow \omega_{x, e}$ and $x \rightarrow \xi\left(x^{-1}, x\right)$ in the vicinity of $e$.

One concludes that $\bar{\omega}$ is a positive normalized linear functional on the involutive algebra $\mathcal{L}_{1}(X, \mathcal{A})$. By continuity it extends to a state of $\mathcal{A} \times_{\xi} X$.

Now let $(\mathcal{H}, \pi, U, \Omega)$ be the GNS-representation of $(\mathcal{A}, X, \sigma)$ induced by $\omega$. Then one has

$$
\begin{aligned}
\bar{\omega}(f) & =\int_{X} \mathrm{~d} x \Delta(x)^{-1} \omega_{x, e}\left(\xi\left(x^{-1}, x\right) f\left(x^{-1}\right)\right) \\
& =\int_{X} \mathrm{~d} x \Delta(x)^{-1}\left(\pi\left(\xi\left(x^{-1}, x\right)\right) \pi\left(f\left(x^{-1}\right)\right) U(x)^{*} \Omega, \Omega\right) \\
& =\int_{X} \mathrm{~d} x(\pi(f(x)) U(x) \Omega, \Omega)
\end{aligned}
$$

A representation $\bar{\pi}$ of $\mathcal{A} \times_{\xi} X$ is now defined by extension of

$$
\bar{\pi}(f)=\int_{X} \mathrm{~d} x \pi(f(x)) U(x), \quad f \in \mathcal{L}_{1}(X, \mathcal{A})
$$

It is obvious that this representation is equivalent with the GNS-representation of $\bar{\omega}$. Conversely, if $\bar{\omega}$ is any state of $\mathcal{A} \times_{\xi} X$ then a state $\omega$ of the covariance system $(\mathcal{A}, X, \sigma)$ can be defined by (9) using the GNS-representation of $\bar{\omega}$ (see the remarks after Theorem 2 of [12]). This representation is then equivalent with the GNS-representation of $\omega$. 


\section{Nonrelativistic particle}

This section is devoted to projective representations occurring in quantum mechanics of a nonrelativistic particle and serves as an example of our approach. It relies heavily on older work by Levy-Leblond [4], work which has been extended by Hagen[7, 8].

The algebra $\mathcal{A}$ is the algebra $\mathcal{C}_{0}\left(\mathbf{R}^{n}\right)$ of classical functions of position. The appropriate group of symmetry transformations is the Galilei group. However, it is easier to start with the subgroup $\mathbf{R}^{n},+$ of shifts. This yields already the standard representation of quantum mechanics. Later on rotations and transformations to a moving frame are added to discuss spin and mass of the particle.

\subsection{Standard representation}

First assume $X=\mathbf{R}^{n},+$. The shifts act as automorphisms of $\mathcal{A}$ by

$$
\left(\sigma_{q} f\right)\left(q^{\prime}\right)=f\left(q^{\prime}-q\right), \quad f \in \mathcal{A}, q, q^{\prime} \in X
$$

A *-representation $\pi$ of $\mathcal{A}$ as bounded operators of $\mathcal{H}=\mathcal{L}_{2}\left(\mathbf{R}^{n}\right)$ is defined by

$$
\pi(f) \psi(q)=f(q) \psi(q), \quad f \in \mathcal{A}, q \in \mathbf{R}^{n}
$$

A unitary representation $U$ of $X$ is defined by

$$
U(q) \psi\left(q^{\prime}\right)=\psi\left(q^{\prime}-q\right), \quad q, q^{\prime} \in \mathbf{R}^{n}
$$

One verifies immediately that $(\mathcal{H}, \pi, U)$ is a representation of $(\mathcal{A}, X, \sigma)$. In particular, any normalized element $\psi$ of $\mathcal{H}$ defines a state of $(\mathcal{A}, X, \sigma)$. The multiplier $\xi$ associated with such a state is identically equal to 1 .

Using Stone's theorem the shift operators can be written as

$$
U(q)=\exp \left(-(i / \hbar) \sum_{j=1}^{n} q_{j} P_{j}\right)
$$

with $P_{j}$ the momentum operators and with $\hbar$ equal to Planck's constant divided by $2 \pi$. A short calculation gives the canonical commutation relations

$$
\left[Q_{j}, P_{k}\right]_{-}=\delta_{j, k} i \hbar
$$


with $Q_{j}$ the multiplication operators

$$
Q_{j} \psi(q)=q_{j} \psi(q)
$$

defined on a suitable domain.

\subsection{Rotation symmetry}

We extend the symmetry group $X$ now to shifts and rotations. We first discuss a particle without spin.

The group law is

$$
\left(q^{\prime}, \Lambda^{\prime}\right)(q, \Lambda)=\left(q^{\prime}+\Lambda^{\prime} q, \Lambda^{\prime} \Lambda\right), \quad q, q^{\prime} \in \mathbf{R}^{n}, \Lambda, \Lambda^{\prime} \in \operatorname{SO}(n)
$$

The representation $\sigma$ is given by

$$
\left(\sigma_{q, \Lambda} f\right)\left(q^{\prime}\right)=f\left(\Lambda^{-1}\left(q^{\prime}-q\right)\right), \quad f \in \mathcal{A}, q, q^{\prime} \in \mathbf{R}^{n}, \Lambda \in \operatorname{SO}(n)
$$

The ${ }^{*}$-representation $(\mathcal{H}, \pi)$ is the standard representation of the previous section. The unitary representation $U$ of $X$ is given by

$$
(U(q, \Lambda) \psi)\left(q^{\prime}\right)=\psi\left(\Lambda^{-1}\left(q^{\prime}-q\right)\right), \quad \psi \in \mathcal{L}_{2}\left(\mathbf{R}^{n}\right), q, q^{\prime} \in \mathbf{R}^{n}, \Lambda \in \operatorname{SO}(n)
$$

Then $(\mathcal{H}, \pi, U)$ is a representation of $(\mathcal{A}, X, \sigma)$. In particular, any normalized element $\psi$ of $\mathcal{H}$ defines a state of $(\mathcal{A}, X, \sigma)$. The multiplier $\xi$ associated with such a state is identically equal to 1 .

\subsection{Spin}

Fix $n=3$. The covariance system $(\mathcal{A}, X, \sigma)$ of the previous section has also states whose associated multiplier $\xi$ is nontrivial. The representation induced by these states is the so-called spinor representation. The reason of their existence is that the group $\mathrm{SO}(3)$ is double connected, with covering group $\mathrm{SU}(2)$.

Given $q \in \mathbf{R}^{3}$, construct the matrix

$$
M(q)=\sum_{j=1}^{3} q_{j} \sigma_{j}
$$


with $\sigma_{1}, \sigma_{2}, \sigma_{3}$ the three Pauli matrices. The matrix $M(q)$ transforms under an element $u$ of $\mathrm{SU}(2)$ into the matrix $M\left(q^{\prime}\right)=u M(q) u^{*}$. It is easy to show that the transformation $q \rightarrow q^{\prime}$ is a rotation, i.e. there exists $\Xi(u) \in \operatorname{SO}(3)$ for which $q^{\prime}=\Xi(u) q$. Note that $\Xi$ is a homomorphism of $\mathrm{SU}(2)$ onto $\mathrm{SO}(3)$. Let $\Lambda \rightarrow v(\Lambda)$ be an inverse of $u \rightarrow \Xi(u)$ in the sense that $\Xi(v(\Lambda))=\Lambda$ for all $\Lambda$ and $v(\Xi(u))=u$ for all $u$ in a neighborhood of the identity matrix. Note that the map $v$ cannot be continuous. A cocycle $\xi$ of $\mathrm{SO}(3)$ is defined by

$$
v(\Lambda) v\left(\Lambda^{\prime}\right)=\xi\left(\Lambda, \Lambda^{\prime}\right) v\left(\Lambda \Lambda^{\prime}\right)
$$

From

$$
\Xi\left(v(\Lambda) v\left(\Lambda^{\prime}\right)\right)=\Lambda \Lambda^{\prime}
$$

and

$$
\Xi\left(\xi\left(\Lambda, \Lambda^{\prime}\right) v\left(\Lambda \Lambda^{\prime}\right)\right)=\Xi\left(\xi\left(\Lambda, \Lambda^{\prime}\right)\right) \Lambda \Lambda^{\prime}
$$

follows that $\Xi\left(\xi\left(\Lambda, \Lambda^{\prime}\right)\right)=\mathbf{I}$. This implies $\xi\left(\Lambda, \Lambda^{\prime}\right)= \pm 1$.

Consider the Hilbert space $\mathcal{H}=\mathcal{L}_{2}\left(\mathbf{R}^{n}\right) \oplus \mathcal{L}_{2}\left(\mathbf{R}^{n}\right)$. An element of this space is denoted $\left(\begin{array}{c}\psi_{1} \\ \psi_{2}\end{array}\right)$. It is normalized if $\left\|\psi_{1}\right\|^{2}+\left\|\psi_{2}\right\|^{2}=1$. $\mathrm{A} *_{-}$ representation $\pi$ of $\mathcal{A}$ is defined by

$$
\pi(f)\left(\begin{array}{c}
\psi_{1} \\
\psi_{2}
\end{array}\right)(q)=f(q)\left(\begin{array}{c}
\psi_{1} \\
\psi_{2}
\end{array}\right)(q)
$$

A projective representation $U$ of $X$ is defined by

$$
U(q, \Lambda)\left(\begin{array}{l}
\psi_{1} \\
\psi_{2}
\end{array}\right)\left(q^{\prime}\right)=v(\Lambda)\left(\begin{array}{l}
\psi_{1} \\
\psi_{2}
\end{array}\right)\left(\Lambda^{-1}\left(q^{\prime}-q\right)\right)
$$

Indeed, one verifies that

$$
\begin{aligned}
& U\left(q^{\prime}, \Lambda^{\prime}\right) U(q, \Lambda)\left(\begin{array}{c}
\psi_{1} \\
\psi_{2}
\end{array}\right)\left(q^{\prime \prime}\right) \\
= & \left.v\left(\Lambda^{\prime}\right) v(\Lambda)\left(\begin{array}{c}
\psi_{1} \\
\psi_{2}
\end{array}\right)\left(\Lambda^{-1}\left(\Lambda^{\prime-1}\right)\left(q^{\prime \prime}-q^{\prime}\right)-q\right)\right) \\
= & \xi\left(\Lambda^{\prime}, \Lambda\right) v\left(\Lambda^{\prime} \Lambda\right)\left(\begin{array}{l}
\psi_{1} \\
\psi_{2}
\end{array}\right)\left(\left(\Lambda^{\prime} \Lambda\right)^{-1}\left(q^{\prime \prime}-q^{\prime}-\Lambda^{\prime} q\right)\right.
\end{aligned}
$$




$$
=\xi\left(\Lambda^{\prime}, \Lambda\right) U\left(q^{\prime}+\Lambda^{\prime} q, \Lambda^{\prime} \Lambda\right)\left(\begin{array}{l}
\psi_{1} \\
\psi_{2}
\end{array}\right)\left(q^{\prime \prime}\right)
$$

Because (11) is satisfied it follows from prop. 2 that $(\mathcal{H}, \pi, U)$ is a representation of $(\mathcal{A}, X, \sigma)$.

Each normalized element $\left(\begin{array}{l}\psi_{1} \\ \psi_{2}\end{array}\right)$ of $\mathcal{H}$ determines a state $\omega$ of $(\mathcal{A}, X, \sigma)$. A tedious calculation yields

$$
\begin{aligned}
\omega_{q, \Lambda ; q^{\prime}, \Lambda^{\prime}}(f)= & \int_{\mathbf{R}^{3}} \mathrm{~d} q^{\prime \prime} f\left(q^{\prime \prime}\right) \\
& \times\left(\begin{array}{c}
\psi_{1} \\
\psi_{2}
\end{array}\right)\left(\Lambda^{\prime} q^{\prime \prime}+q^{\prime}\right) \cdot v\left(\Lambda^{\prime}\right) v(\Lambda)^{*}\left(\begin{array}{c}
\psi_{1} \\
\psi_{2}
\end{array}\right)\left(\Lambda q^{\prime \prime}+q\right)(52
\end{aligned}
$$

In particular,

$$
\omega_{q, \Lambda ; q, \Lambda}(f)=\int_{\mathbf{R}^{3}} \mathrm{~d} q^{\prime \prime} f\left(q^{\prime \prime}\right)\left(\left|\psi_{1}\right|^{2}+\left|\psi_{2}\right|^{2}\right)\left(\Lambda q^{\prime \prime}+q\right)
$$

These diagonal elements of $\omega$ do not reveal whether the state has spin or not. Only correlation effects visible in the off-diagonal elements can reveal the presence of mechanical spin. Consider e.g. a rotation by $\pi$ around the $z$-axis $(\operatorname{denoted} \Lambda)$. Then one has $v(\Lambda)=\sigma_{z}$ so that

$$
\omega_{q, \Lambda ; 0, \mathbf{I}}(f)=\int_{\mathbf{R}^{3}} \mathrm{~d} q^{\prime \prime} f\left(q^{\prime \prime}\right)\left(\bar{\psi}_{1}\left(q^{\prime \prime}\right) \psi_{1}\left(\Lambda q^{\prime \prime}+q\right)-\bar{\psi}_{2}\left(q^{\prime \prime}\right) \psi_{2}\left(\Lambda q^{\prime \prime}+q\right)\right)
$$

The minus sign is a consequence of spin and has the effect that the particle observed in a frame rotated by 180 degrees does not look like expected.

\subsection{Mass}

From now on $X$ is the full Galilei group. Its composition law is

$$
\left(q^{\prime}, \Lambda^{\prime}, t^{\prime}, v^{\prime}\right)(q, \Lambda, t, v)=\left(q^{\prime}+\Lambda^{\prime} q+t v^{\prime}, \Lambda^{\prime} \Lambda, t^{\prime}+t, v^{\prime}+\Lambda^{\prime} v\right)
$$

As before $q \in \mathbf{R}^{n},+$ is a shift operation, and $\Lambda \in \mathrm{SO}(3)$ is a rotation. The remaining parameters describe a transformation to a moving frame with velocity $v \in \mathbf{R}^{n}$ and time $t \in \mathbf{R}$.

For simplicity we consider a particle without spin. Then the relevant

*- representation $\pi$ of $\mathcal{A}$ is the standard representation of section 2.1. The 
Galilei group has nontrivial cocycles $\xi_{\kappa}$ parameterized by some parameter $\kappa \neq 0$ [2, 4]. A possible definition of $\xi_{\kappa}$ is

$$
\xi_{\kappa}\left(q^{\prime}, \Lambda^{\prime}, t^{\prime}, v^{\prime} ; q, \Lambda, t, v\right)=\exp \left(-i \kappa\left[\frac{1}{2} t^{\prime}|v|^{2}+t^{\prime} v^{\prime} \cdot \Lambda^{\prime} v-v^{\prime} \cdot \Lambda^{\prime}(q-t v)\right]\right)
$$

It appears in the following projective representation of $X$

$$
U(q, \Lambda, t, v)=U(q-t v, \Lambda) \exp (i \kappa v \cdot \Lambda Q) \exp \left(i \hbar^{-1} t H\right)
$$

where $H$ is the hamiltonian of the free particle

$$
H=\frac{1}{2 m} P^{2}
$$

the mass $m$ is related to $\kappa$ by $m=\hbar \kappa, P$ and $Q$ are the position and momentum operators (see section 2.1), and $U(q, \Lambda)$ is the unitary representation defined by (44). Indeed, a tedious calculation shows that $U$, defined in this way, satisfies (14) with $\xi=\xi_{\kappa}$. Now (1) can be taken as the definition of the representation $\sigma$ of $X$ as automorphisms of $\mathcal{A}$. By proposition 2 the triple $(\mathcal{H}, \pi, U)$ is a representation of the covariance system $(\mathcal{A}, X, \sigma)$. Any normalized element $\psi$ of $\mathcal{H}$ determines a state $\omega$ of $(\mathcal{A}, X, \sigma)$. The associated $C^{*}$-multiplier is $\xi_{\kappa}$. The state $\omega$ describes a free particle with mass $m=\hbar \kappa$.

\section{Noncanonical commutation relations}

In this section we construct a variant of the model introduced by Doplicher et al [10, 11]. It describes a single quantum particle in spacetime. We do not follow the usual notational conventions of relativity theory. In particular, the inner product $x \cdot y$ is that of $\mathbf{R}^{4}$ and not that of Minkowski space. The reason for doing so is that we use simultaneously two different metric tensors, denoted $g$ resp. $\gamma$.

\subsection{Model}

Let us make the (quite unusual) ansatz that a relativistic particle is characterized by two elements $e$ and $m$ of $\mathbf{R}^{3}$ satisfying $|e|^{2}=|m|^{2}$ and $e \cdot m= \pm 1$. Let $\Sigma \subset \mathbf{R}^{6}$ denote the space of these pairs $(e, m)$. It is by assumption the 
classical configuration space of the particle. See [10, 11] for a motivation of this particular choice. Note that $\Sigma$ is a locally compact manifold in $\mathbf{R}^{6}$. The algebra $\mathcal{A}$ of classical observables equals the $C^{*}$-algebra $\mathcal{C}_{0}(\Sigma)$ of continuous complex functions of $\Sigma$ vanishing at infinity. Consider as symmetry group $X$ of $\Sigma$ the group $\mathbf{R}^{4} \times \mathbf{R}^{4},+$ acting in a trivial way, i.e. $\sigma_{k, q} f=f$ for all $k, q \in \mathbf{R}^{4}$ and $f \in \mathcal{C}_{0}(\Sigma)$. In this way one obtains a covariance system $(\mathcal{A}, X, \sigma)$.

The metric tensor of Minkowski space is denoted $g$. It is the diagonal matrix $[1,-1,-1,-1]$. The geometry of the space $\Sigma$ is described by a metric tensor $\gamma(e, m)$ which is an invertible 4-by-4 matrix with real coefficients depending continuously on $(e, m)$. A possible choice is $\gamma=g$.

Later on the group $X$ will be extended with the Lorentz group. The action of the Lorentz group on functions of $\Sigma$ will be nontrivial. Since integration over $\Sigma$ should be compatible with this action we introduce it already now.

For each $(e, m) \in \Sigma$ form an antisymmetric matrix $\epsilon(e, m)$ by

$$
\epsilon(e, m)=\left(\begin{array}{cccc}
0 & e_{1} & e_{2} & e_{3} \\
-e_{1} & 0 & m_{3} & -m_{2} \\
-e_{2} & -m_{3} & 0 & m_{1} \\
-e_{3} & m_{2} & -m_{1} & 0
\end{array}\right)
$$

The matrix $\epsilon^{-1} \gamma$ will appear often. It transforms positions $q$ into wave vectors $k$. Let us introduce the notation

$$
\eta(e, m)=(e \cdot m) \epsilon^{-1}(e, m) \gamma(e, m)
$$

The factor $(e \cdot m)$, which equals \pm 1 , has been included for physical reasons (one expects that the wave vector $k$ will transform into $g k$ under time reversal, while $q$ transforms into $-g q$ ).

A representation $\sigma$ of the Lorentz group as automorphisms of $\mathcal{C}_{0}(\Sigma)$ is defined by

$$
\sigma_{\Lambda} f(e, m)=f\left(e^{\prime}, m^{\prime}\right) \quad \text { with } \epsilon\left(e^{\prime}, m^{\prime}\right)=\tilde{\Lambda} \epsilon(e, m) \Lambda
$$

The Haar measure of the Lorentz group induces a measure $\rho_{0}$ of $\Sigma$ because each point of $\Sigma$ can be reached by transforming a fixed point, say $e=m=$ $(1,0,0)$. 


\subsection{Quasifree states}

Let $T(e, m)$ be a positive matrix, continuously depending on $(e, m)$, large enough so that $T(e, m)+(i / 2)(e \cdot m) \epsilon(e, m)$ is a positive definite matrix (an appropriate 'covariant' choice of $T$ is discussed later on). Let $w$ be a strictly positive function with integral 1 and let

$$
\mathrm{d} \rho(e, m)=w(e, m) \mathrm{d} \rho_{0}(e, m)
$$

The probability measure $\rho$ together with the choice of $T$ determines a state $\omega$ of $(\mathcal{A}, X, \sigma)$ by

$$
\begin{aligned}
& =\int_{\Sigma}^{\omega_{k, q ; k^{\prime}, q^{\prime}}(f)} \mathrm{d} \rho(e, m) f(e, m) \exp \left(\frac{i}{2}(e \cdot m)(k+\eta q) \cdot \epsilon\left(k^{\prime}+\eta q^{\prime}\right)\right) \\
& \times \exp \left(-\frac{1}{2}\left(k-k^{\prime}+\eta\left(q-q^{\prime}\right)\right) \cdot T\left(k-k^{\prime}+\eta\left(q-q^{\prime}\right)\right)\right)
\end{aligned}
$$

for all $k, k^{\prime}, q, q^{\prime} \in \mathbf{R}^{4}$ The $C^{*}$-multiplier $\xi$ associated with $\omega$ is given by

$$
\xi\left(k, q ; k^{\prime}, q^{\prime}\right)=\exp \left(\frac{i}{2}(e . m)(k+\eta q) \cdot \epsilon\left(k^{\prime}+\eta q^{\prime}\right)\right)
$$

Note that in (63) $k, k^{\prime}$ has the interpretation of a shift in wave vector, $q, q^{\prime}$ that of a shift in position of the particle.

\subsection{Position and momentum operators}

Let $(\mathcal{H}, \pi, U, \Omega)$ be the GNS-representation of $(\mathcal{A}, X, \sigma)$ induced by $\omega$. From the continuity of (63) in $k, k^{\prime}, q, q^{\prime}$ follows, using Stone's theorem, that $U$ has self-adjoint generators. Because $\xi\left(k, q ; k^{\prime}, q^{\prime}\right)$ commutes with $U\left(k^{\prime \prime}, q^{\prime \prime}\right)$ one can write

$$
U(k, q)=\exp (-i k \cdot \gamma Q+i \gamma q \cdot K)
$$

In the context of the GNS-construction the operators $Q_{\mu}$ and $K_{\mu}$ can be written formally as a sum of a multiplication operator and a differential operator, acting on functions $\psi(k, q)$ with value in $\mathcal{C}_{0}(\Sigma)$, From the definition (see the proof of the GNS-construction) follows

$$
U(k, q) \psi\left(k^{\prime}, q^{\prime}\right)=\psi\left(k+k^{\prime}, q+q^{\prime}\right) \xi\left(k^{\prime}, q^{\prime} ; k, q\right)
$$


Comparing expansions of (65) and (66) one obtains

$$
Q_{\mu}=i \sum_{\nu} \gamma_{\mu, \nu}^{-1} \frac{\partial}{\partial k_{\nu}}+\frac{1}{2} q_{\mu}+\frac{1}{2}\left(\eta^{-1} k\right)_{\mu}
$$

and

$$
K_{\mu}=-i \sum_{\nu} \gamma_{\nu, \mu}^{-1} \frac{\partial}{\partial q_{\nu}}+\frac{1}{2} k_{\mu}+\frac{1}{2}(\eta q)_{\mu}
$$

Using these explicit expressions it is easy to calculate the following commutation relations.

$$
\begin{aligned}
{\left[Q_{\mu}, Q_{\nu}\right] } & =-i(e \cdot m)\left(\gamma^{-1} \epsilon \tilde{\gamma}^{-1}\right)_{\mu, \nu} \\
{\left[K_{\mu}, K_{\nu}\right] } & =i(e \cdot m) \epsilon_{\mu, \nu}^{-1} \\
{\left[K_{\mu}, Q_{\nu}\right] } & =-i \gamma_{\nu, \mu}^{-1}
\end{aligned}
$$

Note that the r.h.s. of these expressions is always an operator commuting with all $Q_{\mu}$ and $K_{\mu}$. Commutation relations of this kind have been proposed by Doplicher et al [10, 11] as a simplified model for quantum spacetime.

It is now very easy to calculate physically relevant quantities in the state $\omega$. E.g, one finds $\left(Q_{\mu} \Omega, \Omega\right)=0$ and

$$
\left(Q_{\nu} Q_{\mu} \Omega, \Omega\right)=\int_{\Sigma} \mathrm{d} \rho(e, m)\left[\gamma^{-1}\left(T(e, m)+\frac{i}{2}(e \cdot m) \epsilon(e, m)\right) \gamma^{-1}\right]_{\mu, \nu}(70)
$$

i.e., the state $\omega$ describes a particle with expected position at the origin of spacetime and with variance in position determined by $T \pm(i / 2) \epsilon$.

\subsection{Lorentz transformations}

In what follows we assume that

$$
T\left(e^{\prime}, m^{\prime}\right)=\tilde{\Lambda} T(e, m) \Lambda
$$

with $\left(e^{\prime}, m^{\prime}\right)$ related to $(e, m)$ by (61), i.e. $T$ transforms like $\epsilon$ under Lorentz transformations. Such an assumption is possible. Indeed, let $C$ be any 
positive matrix. Fix a special point $\left(e_{0}, m_{0}\right)$ in $\Sigma$, e.g. the point with $e=$ $m=(1,0,0)$. Use it to define the matrix $\epsilon_{0}$ by $\epsilon_{0}=\epsilon\left(e_{0}, m_{0}\right)$. Now assume that $\tilde{\Lambda} \epsilon_{0} \Lambda=\epsilon_{0}$ implies that $\tilde{\Lambda} C \Lambda=C$. Then $T(e, m)$ is defined by

$$
T(e, m)=\tilde{\Lambda} C \Lambda \quad \text { whenever } \epsilon(e, m)=\tilde{\Lambda} \epsilon_{0} \Lambda
$$

Obviously, $T$ transforms in the same way as $\epsilon$. From

$$
k \cdot T(e, m) k^{\prime}=\Lambda k \cdot C \Lambda k^{\prime}
$$

follows that $T(e, m)$ is positive. In fact we need $T(e, m)+(i / 2)(e \cdot m) \epsilon(e, m) \geq$ 0 . This is satisfied if $C \pm(i / 2) \epsilon_{0} \geq 0$. E.g., $C=(1 / 2) \mathbf{I}$ is a good choice.

If (71) is satisfied then a unitary representation $R$ of the proper Lorentz group is defined by

$$
R(\Lambda) \psi(k, q)(e, m)=\sqrt{\frac{w\left(e^{\prime}, m^{\prime}\right)}{w(e, m)}} \psi\left(\Lambda^{-1} k, \Lambda^{-1} q\right)\left(e^{\prime}, m^{\prime}\right)
$$

with $\left(e^{\prime}, m^{\prime}\right)$ as in (61). One calculates that

$$
R(\Lambda)^{*} \psi(k, q)\left(e^{\prime}, m^{\prime}\right)=\sqrt{\frac{w(e, m)}{w\left(e^{\prime}, m^{\prime}\right)}} \psi(\Lambda k, \Lambda q)(e, m)
$$

It is now straightforward to verify that $R(\Lambda)$ is unitary. One verifies that

$$
\pi\left(\sigma_{\Lambda} f\right)=R(\Lambda) \pi(f) R(\Lambda)^{*}
$$

With the assumption that

$$
\gamma\left(e^{\prime}, m^{\prime}\right)=\tilde{\Lambda} \gamma(e, m) \Lambda
$$

i.e. also $\gamma$ transforms in the same way as $\epsilon$, one obtains

$$
U(\Lambda k, \Lambda q)=R(\Lambda) U(k, q) R(\Lambda)^{*}
$$

Extension of the symmetry group $X$ to include the proper Lorentz group is straightforward. E.g., let

$$
\omega_{k, q, \Lambda ; k^{\prime}, q^{\prime}, \Lambda^{\prime}}(f)=\left(\pi(f) U(k, q)^{*} R(\Lambda)^{*} \Omega, U\left(k^{\prime}, q^{\prime}\right)^{*} R\left(\Lambda^{\prime}\right)^{*} \Omega\right)
$$




\section{Discussion}

We have introduced the notion of a (mathematical) state of a covariance system $(\mathcal{A}, X, \sigma)$. It generalizes the notion of a covariant state of the $C^{*}$ algebra $\mathcal{A}$ by allowing that in the Hilbert space representation the action $\sigma$ of the group $X$ is implemented as a projective representation and by allowing that the cocycle associated with this representation is operator valued (i.e., it is a $C^{*}$-multiplier). For these generalized covariant states we prove a GNStheorem. It can be used as an alternative for working with crossed products of $\mathcal{A}$ and $X$. In fact, the proof captures all essential elements of the proof of the existence of the crossed product algebra. The shift in emphasis from crossed product algebra to states of a covariance system has many advantages. In particular, the problem that the crossed product algebra depends on the $C^{*}$ multiplier is circumvented. E.g., mass and spin of a quantum particle are properties of states of a covariance system, involving different $C^{*}$-multipliers. In the more traditional approach particles with or without spin are described by states on different $C^{*}$-algebras.

A limitation of the present work is that representations involving antiunitary operators are not included. Such representations are essential for physical applications. Wigner [1] showed that all symmetry elements appearing in quantum mechanics must be implemented either by unitary or anti-unitary operators of the Hilbert space of wave functions. These antiunitary operators appear only in case of discrete symmetries. Indeed, one can always assume that the neutral element of the symmetry group is implemented as the identity operator. Then any element which is continuously connected with the neutral element must also be implemented as a unitary operator. We think that the omission of anti-unitary representations can be handled easily in most situations. It is clear from the examples that the physically relevant representation of the covariance system is usually already fixed by considering a subgroup of symmetries (e.g., the standard representation of quantum mechanics is already obtained by considering only the subgroup of spatial shifts). In such cases the anti-unitary implementation of discrete symmetry elements can be added 'by hand', i.e. without relying on the GNS-theorem.A good example[3] is time reversal symmetry, denoted $\theta$. When added to the Galilei group it anti-commutes with velocity $v$ and time $t$, and commutes with position $q$ and rotation $\Lambda$. It is implemented as the anti-unitary operator which maps each wave function onto its complex conjugate. In the relativistic example $\theta=-g$ is an element of the full Poincaré 
group. Its implementation is

$$
R(\theta) \psi(k, q)(e, m)=\sqrt{\frac{w(-e, m)}{w(e, m)}} \overline{\psi(g k, \theta q)(-e, m)}
$$

The section on non-relativistic quantum mechanics picks up old ideas about the role of covariance systems in quantum mechanics and illustrates our approach to quantum mechanics and quantum field theory. It does not involve a quantization step referring to classical mechanics. It starts from a $C^{*}$-algebra $\mathcal{A}$ of 'classical' functions which are accessible for experimental measurement. A group $X$ of symmetries, acting as automorphisms of $\mathcal{A}$, is considered. Together they form a covariance system. E.g., if $\mathcal{A}$ is a $C^{*}$ algebra of functions of position in $\mathbf{R}^{3}$ and $X$ is the Galilei group then the standard representation of quantum mechanics is a (projective) representation of the covariance system $(\mathcal{A}, X, \sigma)$.

The third section about the model of quantum spacetime has been added to give an example of projective representations involving operator valued cocycles i.e. non-trivial $C^{*}$-multipliers. The immediate effect of these are non-canonical commutation relations. Another aspect, well illustrated by this example, is how the GNS-construction can be used to build physically interesting representations starting from an explicit expression for a state of the covariance system. It is quite clear that our treatment of this model is far from complete. A full study is out of scope of the present paper and will be reported elsewhere.

\section{Acknowledgements}

This work benifited from financial support by the Bilateral Scientific and Technological Cooperation Programme between Poland and Flanders and by the Research Council of the University of Antwerp. 


\section{References}

[1] E.P. Wigner, Gruppentheorie und ihre Anwendung auf die Quantenmechanik der Atomspektren (Braunschweig, 1931); Group theory and its applications to the quantum mechanics of atomic spectra (Academic press, New York, 1959).

[2] V. Bargmann, On unitary representations of continuous groups, Ann. Math. 59, 1-46 (1954).

[3] J. Schwinger, The Theory of Quantized Fields. I, Phys. Rev. 82, 914-927 (1951).

[4] J.-M. Levy-Leblond, Galilei group and nonrelativistic quantum mechanics, J. Math. Phys. 4, 776-788 (1963).

[5] S. Doplicher, D. Kastler, D.W. Robinson, Covariance Algebras in Field Theory and Statistical Mechanics, Commun. Math. Phys. 3, 1-28 (1966).

[6] R.C. Busby, H.A. Smith, Representations of twisted group algebras, Trans. Amer. Math. Soc. 149, 503-537 (1970).

[7] C.R. Hagen, The Bargmann-Wigner Method in Galilean Relativity, Commun. Math. Phys. 18, 97-108 (1970).

[8] C.R. Hagen, Galilean invariant Lee model for all spins and parities, Commun. Math. Phys. 21, 219-236 (1971).

[9] M. Takesaki, Theory of operator algebras, I (Springer-Verlag, 1979), Ch V, Sect 7 .

[10] S. Doplicher, K. Fredenhagen, J.E. Roberts, Spacetime quantization induced by classical gravity, Phys. Lett. B331, 39-44 (1994)

[11] S. Doplicher, K. Fredenhagen, J.E. Roberts, The Quantum Structure of Spacetime at the Planck Scale and Quantum Fields, Commun. Math. Phys. 172, 187-220 (1995)

[12] J. Naudts, $C^{*}-$ Multipliers, crossed product algebras, and canonical commutation relations, math-ph/9907008. 